\title{
MONITORAMENTO DA AUTENTICIDADE DE AMOSTRAS DE BEBIDAS ALCOÓLICAS ENVIADAS AO INSTITUTO ADOLFO LUTZ EM SÃO PAULO' ${ }^{1}$
}

\author{
Leticia Araujo Farah NAGATO ${ }^{2},{ }^{,}$, Maria Cristina DURAN², Miriam Solange Fernandes CARUSO ${ }^{3}$, \\ Roberto Carlos Fernandes BARSOTTI², Elza Schwarz Gastaldo BADOLATO ${ }^{3}$
}

\begin{abstract}
RESUMO
Este trabalho teve como objetivo verificar a autenticidade de bebidas alcoólicas (whisky, vodka, conhaque de gengibre, etc.) enviadas para análise ao Instituto Adolfo Lutz no período de 1993 a agosto de 1999. Devido à presença no mercado nacional de bebidas alcoólicas clandestinas, principalmente aquelas com alto valor agregado, foi realizado o monitoramento através da análise de composição química destas bebidas. Normalmente estes produtos são elaborados com álcool, água, aroma e corante caramelo e que por falta de controle dessas matérias-primas, podem oferecer risco potencial à saúde humana pela presença de metanol. A técnica empregada na análise dos componentes secundários e metanol das amostras foi realizada através da cromatografia em fase gasosa com detector de ionização de chama. Observou-se que em 608 amostras de bebidas alcoólicas analisadas, 391 eram falsificadas e dentre estas 2 apresentaram teores de metanol acima do limite tolerado $(200 \mathrm{mg} / 100 \mathrm{~mL}$ de álcool anidro) pela legislação em vigor. Os resultados obtidos sugerem uma grande persistência em se produzir bebidas alcoólicas falsificadas. Desta forma, é muito importante estar sempre monitorando estes produtos e identificando os diferentes tipos de falsificações existentes no país.
\end{abstract}

Palavras-chave: bebidas alcoólicas; autenticidade; cromatografia gasosa; componentes secundários; metanol.

\section{SUMMARY}

EVALUATION OF AUTHENTICITY OF ALCOHOLIC BEVERAGE SAMPLES EXAMINED BY THE INSTITUTO ADOLFO LUTZ (SÃO PAULO, BRAZIL). This work aimed at verifying the authenticity of alcoholic beverages (whisky, vodka, ginger spirit, etc.) sent for analysis by the Instituto Adolfo Lutz, São Paulo from 1993 to August 1999. The chemical composition of these drinks was studied because of the presence of illicit alcoholic beverages on the national market, especially those with a high commercial value. These products are usually elaborated with alcohol, water, flavor and caramel coloring. Since there is no control over these raw materials, these drinks could offer a potential risk to human health because of the presence of methanol. The method employed in the analysis of congeners and methanol in alcoholic products was gas-liquid chromatography with flame ionization detector. It was observed that 391 of the 608 samples of alcoholic beverages analyzed, were counterfeit, and 2 of them presented levels of methanol above the limit tolerated by the Brazilian legislation $(200 \mathrm{mg} / 100 \mathrm{~mL}$ absolute alcohol). The results obtained suggest a great persistence in producing counterfeit alcoholic beverages. Thus, it is very important to constantly analyze these products, and identify the different kinds of counterfeiting in the country.

\footnotetext{
${ }^{1}$ Recebido para publicação em 28/03/00. Aceito para publicação em 18/04/01.

${ }^{2}$ Seção de Bebidas, Instituto Adolfo Lutz - Av. Dr. Arnaldo, 355, CEP 01246-902 - S. Paulo, SP.

${ }^{3}$ Laboratório de Cromatografia, Inst. Adolfo Lutz.

* A quem a correspondência deve ser enviada.
}

Keywords: alcoholic beverages; authenticity; gas-chromatography; congeners; methanol.

\section{1 - INTRODUÇÃO}

Falsificar significa alterar, contrafazer, imitar uma substância que o autor propôs igualar em aparência, tornando-a externamente semelhante a original, como define o Código Penal Brasileiro.

A falsificação de bebida alcoólica ocorre principalmente em bebidas com alto valor comercial como por exemplo, os whiskies importados. Entretanto, whiskies nacionais, vodkas, conhaques de gengibre e aguardentes de cana são, também passíveis de falsificação, pois apesar de apresentarem menor valor agregado, o consumo destas bebidas pela população é elevado.

As bebidas alcoólicas falsificadas normalmente são elaboradas, em sua maioria, através da simples adição de água às bebidas autênticas, ou ainda, por mistura de água, álcool (para fins alimentícios, ou não), aroma e corante caramelo [2].

As matérias-primas utilizadas neste processo não têm nenhum tipo de controle, e portanto, a ingestão destas bebidas pode oferecer um risco potencial à saúde humana, principalmente, pela presença do metanol e/ ou de outras substâncias em níveis acima dos limites tolerados pela legislação em vigor $[2,9,11]$.

Durante todo o processo de fabricação de bebida alcoólica são formados diversos compostos, dentre eles aldeídos, álcoois, ácidos e ésteres, denominados componentes secundários. Tais compostos, embora presentes em baixas concentrações (abaixo de $0,5 \%$ ), são importantes na determinação da qualidade do produto final $[4,5]$.

O metanol é um constituinte naturalmente presente nas bebidas alcoólicas, em quantidades inferiores aos demais componentes. Nas bebidas clandestinas, em virtude de eventual mistura com álcool não potável, podem ser encontrados valores de metanol bem acima do limite tolerado pela legislação brasileira que é de $200 \mathrm{mg} / 100 \mathrm{~mL}$ de álcool anidro para aguardentes, whiskies e outras bebidas destiladas; e de $400 \mathrm{mg} / 100 \mathrm{~mL}$ de álcool anidro para conhaques e aguardentes de frutas [5].

A ingestão de bebidas com concentrações elevadas de metanol pode causar intoxicação, que varia desde dor de cabeça, náuseas e vômitos até cegueira e morte. Alguns autores consideram que o consumo de $20 \mathrm{~mL}$ de metanol pode provocar cegueira e que $60 \mathrm{~mL}$ constitui dose letal $[3,7]$. 
Dados da literatura apontam que o teor máximo de metanol obtido no processo de fabricação dos diferentes tipos de bebidas alcoólicas pode atingir até $1 \mathrm{~g} / 100 \mathrm{~mL}$ de álcool anidro [10], o que sugere tratar-se de fraudes, bebidas com concentrações de metanol acima deste valor.

Diante do exposto, o Instituto Adolfo Lutz, que é um Laboratório de Saúde Pública, mantém a preocupação em monitorar as bebidas comercializadas no Estado de São Paulo, a fim de avaliar a qualidade das mesmas, bem como, alertar as autoridades competentes e a população quanto aos prováveis riscos que as bebidas clandestinas podem causar.

É grande a diversidade de amostras de bebidas alcoólicas que são encaminhadas ao Instituto Adolfo Lutz para verificação de autenticidade, pelos órgãos como: Polícia Civil de S. Paulo, Defesa do Consumidor (DECON e PROCON) e Vigilância Sanitária do Estado de São Paulo.

Este trabalho teve como objetivo verificar a autenticidade de bebidas alcoólicas (whisky, vodka, aguardente, conhaque de gengibre e outras) enviadas para análise ao Instituto Adolfo Lutz, no período de abril de 1993 a agosto de 1999.

\section{2 - MATERIAIS E MÉTODOS}

Foram analisadas 608 amostras de vários tipos de bebidas alcoólicas, do período de abril de 1993 a agosto de 1999, conforme a Tabela 1.

TABELA 1: Tipos de bebidas alcóolicas analisadas e quantidade

\begin{tabular}{cc}
\hline Tipo & o de amostras \\
\hline Whisky nacional & 141 \\
Whisky importado & 266 \\
Vodka & 71 \\
Conhaque & 4 \\
Conhaque de gengibre & 41 \\
Amargo & 16 \\
Aguardente-de-cana & 46 \\
Rum & 2 \\
Aperitivo & 6 \\
Licor & 8 \\
Gim & 2 \\
Tequila & 5 \\
\hline Total & $\mathbf{6 0 8}$ \\
\hline
\end{tabular}

\section{1 - Materiais}

\subsection{1 - Equipamentos}

Cromatógrafo a gás com detector de ionização de chama, marca - Finnigan 9001; equipado com coluna empacotada 5\% Carbowax 20M, suporte Carbopack B, $2 \mathrm{~m} \times 2 \mathrm{~mm}$, da CG Analítica.

Densímetro digital automático, marca - Mettler, calibrado a $20^{\circ} \mathrm{C}$.

\subsection{2 - Reagentes e solventes}

Padrões cromatográficos: acetaldeído, metanol, acetato de etila, n-propanol, isobutanol, n- butanol, 2- metil-butanol e 3-metil -butanol, 3-pentanol (padrão interno). Foram utilizados padrões de marca - Sigma.

Solvente: álcool etílico grau cromatográfico, marca - Merck.

A solução de calibração dos padrões para a quantificação do metanol e componentes secundários foi feita através da mistura de acetaldeído, metanol, acetato de etila, n-propanol, isobutanol, n- butanol, 2-metil-butanol e 3-metil-butanol nas concentrações de cerca de: 9, 17, 20, 16, 25, 4, 7 e 20mg/100mL respectivamente em solução de álcool etílico a $40 \%$ v/v. Foi adicionado a essa solução de calibração 3-pentanol como padrão interno, a uma concentração final de $20 \mathrm{mg} / 100 \mathrm{~mL}$. Para a amostra de bebida alcoólica também foi adicionado padrão interno na mesma concentração.

\section{2 - Métodos}

\subsection{1 - Preparo da amostra}

As amostras de bebidas destiladas foram injetadas diretamente no cromatógrafo. As bebidas alcoólicas por mistura foram destiladas conforme o método oficial de grau alcoólico [6], antes da injeção no cromatógrafo.

A avaliação das amostras de bebidas alcoólicas com suspeita de falsificação foi feita comparativamente com as bebidas autênticas de mesma marca e fabricante, através da determinação do teor de álcool etílico (\%v/v) e dos componentes secundários $(\mathrm{mg} / 100 \mathrm{~mL})$ característicos de cada tipo de produto. Também foi feita a análise de odor próprio de cada bebida.

As bebidas autênticas foram fornecidas pelos respectivos fabricantes, ou por seus representantes legais, no caso das importadas.

TABELA 2. Parâmetros cromatográficos

\begin{tabular}{cc}
\hline Vazão do gás de arraste $\left(\mathrm{N}_{2}\right)$ & $25 \mathrm{~mL} / \mathrm{min}$ \\
Vazão do ar sintético & $175 \mathrm{~mL} / \mathrm{min}$ \\
Vazão do hidrogênio & $15 \mathrm{~mL} / \mathrm{min}$ \\
Volume de injeção & $1,0 \mu \mathrm{L}$ \\
Temperatura do injetor & $200^{\circ} \mathrm{C}$ \\
Temperatura do detector & $250^{\circ} \mathrm{C}$ \\
Programação de temperatura do forno & $70^{\circ} \mathrm{C}$ por $2 \mathrm{~min}, 6^{\circ} \mathrm{C} / \mathrm{min}$ \\
& até $133^{\circ} \mathrm{C}$, e $3^{\circ} \mathrm{C} / \mathrm{min}$ \\
& até $160^{\circ} \mathrm{C}(2 \mathrm{~min})$ \\
\hline
\end{tabular}

1. Determinação de álcool etílico em volume: foi determinada a densidade relativa de cada amostra e convertida à graduação alcoólica, segundo métodos oficiais do Ministério da Agricultura [6] e métodos analíticos da A. O. A. C., [1].

2. Determinação de metanol e componentes secundários: foi empregada a técnica de cromatografia em fase gasosa com detector de ionização de chama se- 
gundo método descrito na literatura por MARTIN et al [8], adaptado às nossas condições de trabalho conforme Tabela 2. Para a quantificação de metanol e componentes secundários na bebida foi utilizado o método de padronização interna com 3-pentanol.

\section{3 - RESULTADOS E DISCUSSÃO}

Previamente as amostras foram injetadas no cromatógrafo a gás, sem a adição de padrão interno, para uma avaliação qualitativa de suas composições. O mesmo procedimento foi adotado para as respectivas amostras autênticas. Deste modo, os perfis cromatográficos foram comparados, observando-se se havia diferenças entre eles quanto à presença dos componentes secundários.

A Figura 1 ilustra os cromatogramas obtidos de uma amostra de whisky autêntico importado (1a) e de uma amostra de whisky falsificado (1b).

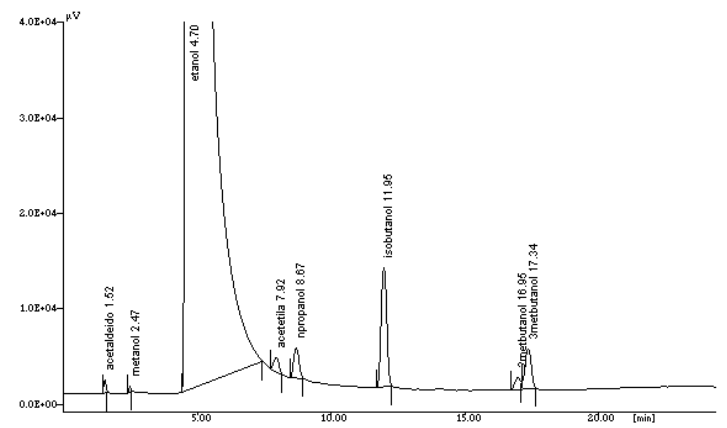

1 a. Whisky autêntico importado

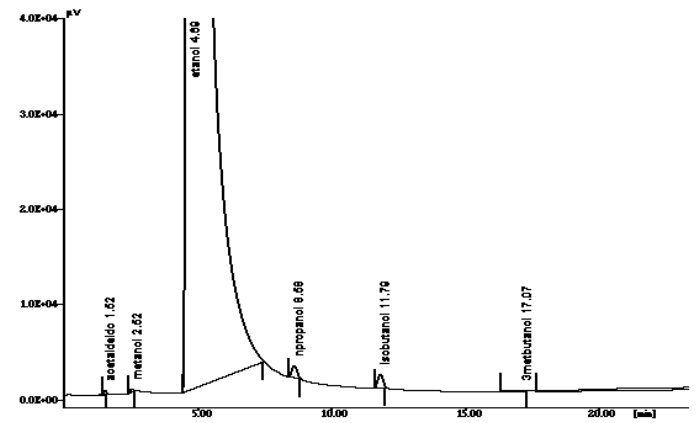

1 b. Whisky falsificado

FIGURA 1. Cromatogramas de whiskies por cromatografia em fase gasosa com detector de ionização de chama, coluna empacotada $5 \%$ Carbowax $20 \mathrm{M}(2 \mathrm{~m} \times 2 \mathrm{~mm})$.

Observando-se os 2 cromatogramas, nota-se no primeiro as presenças de etanol e dos compostos: acetaldeído, metanol, acetato de etila, e álcoois superiores n-propanol, isobutanol, 2 e 3 metil-butanol, em proporções adequadas para uma amostra autêntica; já no segundo cromatograma (Figura 1b), verifica-se a presença de etanol, uma pequena proporção de acetaldeído e metanol e uma quantidade muito reduzida de álcoois superiores. A diferença dos perfis cromatográficos de ambas amostras é notória, o que demonstra uma falsificação no segundo caso.
A Figura 2 ilustra os cromatogramas obtidos em uma análise quantitativa de amostras de whisky autêntico (2a), e falsificado (2b), com adição de padrão interno.

A determinação quantitativa de metanol e componentes secundários (ou compostos voláteis não álcool) é feita rotineiramente pelo Instituto Adolfo Lutz, sempre que é solicitada, ou quando a análise qualitativa apresenta dúvidas.

Os demais tipos de bebidas alcoólicas autênticas que foram analisadas apresentam cromatogramas similares aos da Figura 1a, contudo a proporção de componentes secundários é própria de cada um.

Quanto à análise de grau alcoólico observou-se que as bebidas falsificadas possuíam valores diferentes quando comparadas com amostras de bebidas alcoólicas autênticas, sendo que na maioria das vezes este teor estava abaixo do limite mínimo exigido pelo Decreto 2314/97 do Ministério da Agricultura [5].

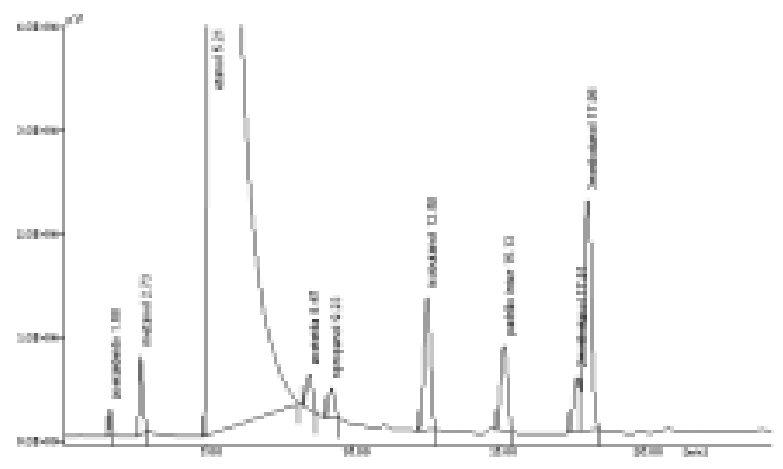

2 a. Whisky autêntico com adição de padrão interno

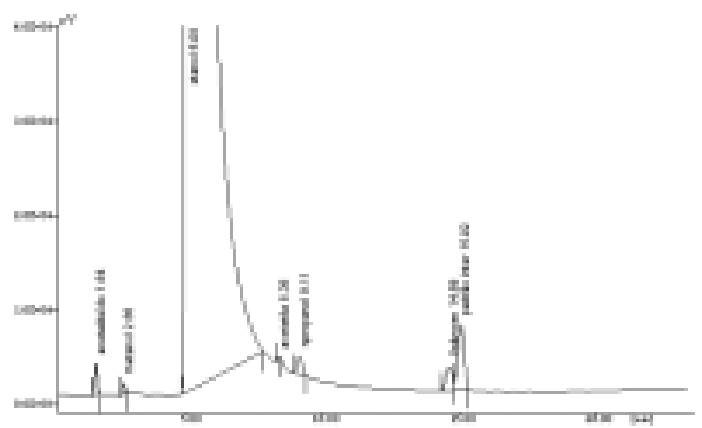

2 b. Whisky falsificado com adição de padrão interno

FIGURA 2. Cromatogramas de whiskies por cromatografia em fase gasosa com detector de ionização de chama, análise quantitativa. Tempo de retenção do padrão interno (3-pentanol) $15 \mathrm{~min}$.

De um modo geral, a análise qualitativa foi suficiente para se afirmar que as bebidas eram falsificadas, pois houveram diferenças grandes entre os perfis cromatográficos destas e das autênticas. Neste monitoramento não houve necessidade de se fazer a análise quantitativa, pois as falsificações eram muito evidentes, e os componentes secundários encontravam-se 
em proporções bem pequenas, ou nem estavam presentes nas amostras.

A Figura 3 mostra que 391 amostras (64\%) das 608 bebidas alcoólicas analisadas estavam falsificadas.

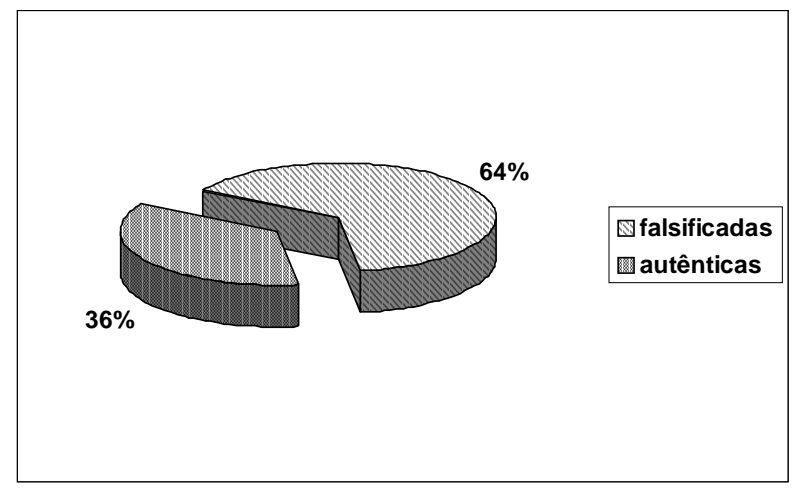

FIGURA 3. Percentual de amostras falsificadas sobre o total de bebidas alcoólicas analisadas.

A Tabela 3 ilustra as freqüências das amostras falsificadas e aprovadas por tipo de produto analisado.

TABELA 3. Número de amostras analisadas por tipo de bebida

\begin{tabular}{|c|c|c|c|c|}
\hline Tipodeproduto & Total & Aprovadas & Falsificadas & $\%$ Falsif \\
\hline Whisky nacional & 141 & 27 & 114 & 81 \\
\hline Whisky importado & 266 & 108 & 158 & 59 \\
\hline Vodka & 71 & 25 & 46 & 65 \\
\hline Conhaque & 4 & 1 & 3 & 75 \\
\hline Conhaque de gengibre & 41 & 8 & 33 & 80 \\
\hline A m argo & 16 & 2 & 14 & 88 \\
\hline Aguardente-de-cana & 46 & 26 & 20 & 43 \\
\hline Rum & 2 & 2 & 0 & -. \\
\hline A peritivo & 6 & 4 & 2 & 33 \\
\hline Licor & 8 & 7 & 1 & 13 \\
\hline Gim & 2 & 2 & 0 & -- \\
\hline Tequila & 5 & 5 & 0 & -. \\
\hline Total & 608 & 217 & 391 & \\
\hline
\end{tabular}

Observou-se que dentre os diversos tipos de bebidas alcoólicas, o whisky (114 amostras de marcas nacionais e 158 de marcas importadas) foi o que apresentou a maior quantidade de falsificação, seguido da vodka (46 amostras), conhaque de gengibre (33 amostras) e amargo (14 amostras falsificadas). Já a aguardente-de-cana teve um número equivalente entre as amostras consideradas falsificadas e autênticas (conforme a Tabela 3). As demais bebidas alcoólicas foram aprovadas em sua maioria.

Dentre as 391 amostras reprovadas verificou-se que 2 amostras clandestinas apresentaram elevado teor de metanol, 1 de vodka procedente de São Paulo (com $14 \mathrm{~g} / 100 \mathrm{~mL}$ ), e 1 de aguardente-de-cana produzida na Bahia (com $10 \mathrm{~g} / 100 \mathrm{~mL}$ ), sendo que as mesmas causaram intoxicação humana aguda grave (cegueira).

\section{CONCLUSÕES}

1. A partir dos resultados experimentais obtidos observou-se que $64 \%$ das amostras analisadas foram classificadas como falsificadas, o que sugere uma persistência do comércio de bebidas clandestinas.

2. O whisky representou $70 \%$ do total das amostras de bebidas falsificadas, mostrando que a falsificação é mais freqüente em produtos com alto preço de mercado.

3. Um outro aspecto observado é que tem aumentado o número de bebidas clandestinas com rótulo de bebidas alcoólicas nacionais que apresentam menor valor comercial.

4. O comércio de bebidas alcoólicas clandestinas além do aspecto ilegal, apresenta um sério risco à população, pois estas bebidas podem conter substâncias nocivas à saúde.

\section{REFERÊNCIAS BIBLIOGRÁFICAS}

[1] ASSOCIATION OF OFFICIAL ANALYTICAL CHEMISTS. Official Methods of Analysis of A.O.A.C. $16^{\text {th }}$ ed., Gaithersburg, 1995. V.II, cap. 26, p.4

[2] BADOLATO, E.S.G., AUED-PIMENTEL, S., DURAN, M.C., NAGATO, L.A.F. Falsificação de uísques no Estado de São Paulo. Bol. IAL, São Paulo, v. 3, n. 1, p. 2, 1993.

[3] BINDLER, F., VOGES, E., LAUGEL, P. The problem of methanol concentration admissible in distilled fruit spirits. Food Add. Contam., London, v. 5, n. 3, p. 343-351, 1988.

[4] BOZA, Y., HORII, J. A destilação na obtenção da aguardente de cana de açúcar. Bol. SBCTA, Campinas, v. 33, n. 1, p. 98-105, 1999.

[5] BRASIL. Leis, Decretos, etc. Decreto no. 2314 de 4 de set de 1997. Diário Oficial da União, Brasília, n. 171, 5 de set de 1997. Seção I, p.19556. [Regulamenta a Lei no. 8918, de 14-07-1994, que dispõe sobre a padronização, a classificação, o registro, a inspeção, a produção e fiscalização de bebidas].

[6] BRASIL. Leis, Decretos, etc. Portaria no. 76 de 27 de nov. de 1986 do Min. Da Agricultura. Diário Oficial da União, Brasília, n. 232, 3 de dez. de 1986. Seção I, p.18153. [Aprova os métodos analíticos, que passam a constituir padrões oficiais para análise de bebidas e vinagres, na forma estabelecida pelo Decreto no. 73267, de 06-12-1973].

[7] GOSSELIN, R.E., HODGE, H.C., SMITH, R.P., GLEASON, M. N. Clinical Toxicology of Commercial Products, The Williams \& Wilkins Co., Baltimore, 1976, p. 229-233.

[8] MARTIN, G. E., BURGGRAFF, J. M., DYER, R. H., BUSCEMI, P. C. Gas-liquid chromatographic determination of congeners in alcoholic products with confirmation by gas chromatography / mass spectrometry. J. Assoc. Off. Anal. Chem., Washington, v. 64, n. 1, p. 186-190, 1981.

[9] MIRANDA, M.P., DANTAS, V. P., DEL CORRAL, F. S. D. Grau alcoólico e níveis de metanol em aguardentes do comércio (no Estado da Bahia). Bol SBCTA, Campinas, v. 26, n. 2, p.104-107, 1992.

[10] SIMPKINS, W. A. Congener profiles in the detection of illicit spirits. J. Sci. Food Agric., London, v. 36, p. 367-376, 1985

[11] ZENEBON, O., BADOLATO, E. S.G., NAGATO, L. A. F., DURAN, M. C., AUED-PIMENTEL, S., VASCONCELOS, D. S. A. Metanol - Avaliação da ocorrência de intoxicações causadas pela ingestão de bebidas alcoólicas no Estado de São Paulo. Bol. SBCTA, Campinas, v. 30, n. 1, p. 71-74, 1996. 